\section{Determination of the Solid Volume Percentage below Detectable Size in Electronic Counter Size Analysis}

SINCE we proposed a graphical procedure to estimate the solid volume percentage below detectable size ${ }^{1}$, an alternative method has been suggested by Eckhoff ${ }^{2}$. Using our data, he obtained two values for tho solid volume, $V_{\infty}$, which are about 5 per cent below our estimate. The purpose of this communication is to discuss the reasons for these differences. The numerical differences arise through different extrapolation criteria; thus values similar to Eckhoff's can be obtained from our method with his criteria. Our value, however, cannot be found from Eckhoff's mothod. This is not a criticism; his method was not intended for use with an equation.

When our results are plotted on a sufficiently large scale, the reasons for the different estimates of $V_{\infty}$ become clear (Fig. 1). The average straight line through the five points (Fig. 1, line $A$ ) gives $V_{\infty}=20,600$. This is in accordance with a Rosin-Rammler distribution. Eckhoff obtains $V_{\infty}=19,259$ and 19,62 I by two different extrapolations through the finest size region of a histogram. 19,259 results from a linear extrapolation from the three finest fractions intercepting the size axis at about 2 microns. Insufficient experimental evidence precludes questioning the value 19,259. The method of reaching it, however, is wrong because the sample contained material below 2 microns. $V_{\infty}=20,000$ can be obtained from Fig. 1 by drawing a line through the three finest fractions; this is not shown to avoid congestion. A line through the two finest fractions (Fig. 1, line B) gives $V_{\infty}=19,600$. As with Eckhoff's method, these lower values arise through extrapolation from the finest region only. Thus, although our method was explained in terms of size distribution equations, their application is not necessary; this was noted in our penultimate para$\operatorname{graph}^{1}$.

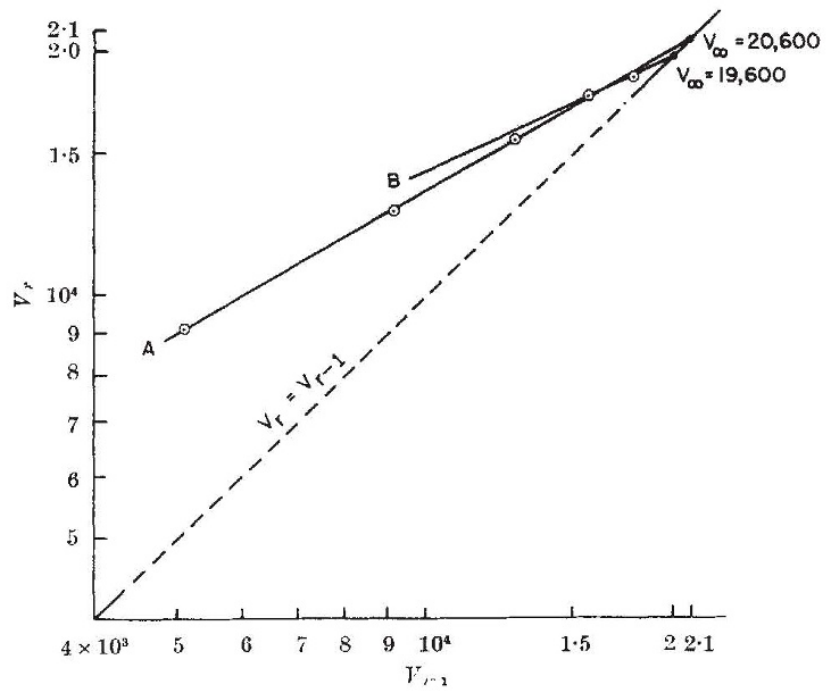

Fig. 1. (Xraphical determination of $V \propto$ (data from ref. 1 ). $A$, Average line through five points (Rosin-Rammler distribution); $B$, line through

Two issues concerning size distribution equations have been raised. First, have they a physical rather than a statistical foundation and, second, even with a physical basis, can tho behaviour of quantities in the measured Jange be assumed to extend into the undetectable part of the distribution? We have already pointed out that there is no assurance that the law outside the measured range is the same as inside, so that the results of this extrapolation technique must be used with discretion ${ }^{1}$. Only an experi- mental method would avoid these difficulties, and an elegant procedure ${ }^{3}$ is now available.

There is growing evidence that several equations of size distribution, once regarded as empirical, have a sound physical foundation ${ }^{4}$. Additionally, recent measurements ${ }^{5}$ have demonstrated the applicability of these equations down to $3 \times 10^{-2}$ microns. These considerations, together with a knowledge of the method of sample preparation (batch grinding), suggested that a Rosin-Rammle! distribution was more likely than not; thus, $V_{\infty}=$ 20,600 . Without this information, however, we would propose $V_{\infty}=19,600$, at the same time pointing out the strong evidence for a Rosin-Rammler distribution.

Eckhoff illustrated the results of the three $V_{\infty}$ estimates on a $\log$ probability plot, showing that $V_{\infty}=19,259$ gave a straight line for the finest 60 per cent of the material. On a Rosin-Rammler plot (log log reciprocal versus $\log$ ) $V_{\infty}=19,259$ gives a strong curve, less curvature is produced by $V_{\infty}=19,600$, while $V_{\infty}=20,600$ results in a straight line.

We thank Mr. R. K. Eckhoff for his discussion.

C. C. Harris

Henry Krumb School of Mines,

Columbia University,

New York.

A. Jowet'

The University,

Leeds 2.

${ }^{1}$ Harris, C. C., and Jowett, A., Nature, 208, 175 (1965).

2 Eckhoff, R. K., Nature, 210, 765 (1966).

${ }^{3}$ Sopp, O. I., and Moum, J., Nature, 210, 724 (1966).

- Harris, (. C., Trans. Inst. Min. Metall., 75, C37 (1966).

${ }^{5}$ Kondo, Y., and Gaudin, A. M., Trans. Amer. Inst. Min. Eng., 229, $29 \%$ (1964).

\section{Coincidence Error in Coulter Counter Particle Size Analysis}

Mathematical expressions for the count loss that occurs when two or more particles enter the orifice of a Coulter counter in close succession are usually based on the concept of a sensing zone within which two or more: particles cannot be counted separately ${ }^{1-3}$. Thus, Princen and $\mathrm{Kwolek}{ }^{3}$ give an expression for count loss (primary coincidence) in a suspension of particles of equal size.

$$
n=N-c N^{2}
$$

where $N$ is the real number of particles in volume $V$, $n$ is the observed number, and $c=A / 2 V$, where $A$ is the volume of the sensing zone.

Assuming that particles which coincide in the sensing zone produce a voltage pulse equal in height to the sum of the individual heights and contribute a count gair (secondary coincidence) to the observed counts at higher thresholds, and assuming a log-normal distribution of particle size, Princen and Kwolek developed theoretical expressions relating the observed count, the real number of particles, and the distribution parameters. Their equations are not easy to solve, even with a computer, and their assumptions are not necessarily valid in practice.

Equation 1, however, gives a clue to the possibility of correcting for both primary and secondary coincidenec: by an empirical mothod that involves no assumptions about size distribution or pulse summation.

When a primary suspension is diluted, the number of particles per unit volume is inversely proportional to the dilution factor, and equation 1 can be written as

$$
n / C=1-c C
$$

where $C$ is the eonecntration relative to the primary suspension. Thus, $n / C$ should be linear with $C$, and extrapolation should give $n / C_{0}=N$ in the primary suspension. If in practice the plot is linear for uniform particles of threshold size, it should still be lincar for the 